\title{
Numerical investigation of floating breakwater movement using SPH method
}

\author{
A. Najafi-Jilani ${ }^{1}$ and A. Rezaie-Mazyak ${ }^{2}$ \\ ${ }^{1}$ Civil Engineering, Islamic Azad University, Islamshahr Branch, Iran \\ ${ }^{2}$ Civil Engineering, Tarbiat Modares University, Tehran, Iran
}

\begin{abstract}
In this work, the movement pattern of a floating breakwater is numerically analyzed using Smoothed Particle Hydrodynamic (SPH) method as a Lagrangian scheme. At the seaside, the regular incident waves with varying height and period were considered as the dynamic free surface boundary conditions. The smooth and impermeable beach slope was defined as the bottom boundary condition. The effects of various boundary conditions such as incident wave characteristics, beach slope, and water depth on the movement of the floating body were studied. The numerical results are in good agreement with the available experimental data in the literature The results of the movement of the floating body were used to determine the transmitted wave height at the corresponding boundary conditions
\end{abstract}

KEY WORDS: Floating Breakwater; Smoothed Particle Hydrodynamics(SPH); Paddle Wavemaker; Regular Waves

\section{INTRODUCTION}

Floating breakwater is generally used to maintain tranquility in port. It is also used frequently in marine works, military operations, fishery activities, recreational ports etc. Navigation and harboring of ships in many ports are significantly affected by influx of waves. Hence, floating breakwater is necessary to reduce wave heights in a specific location to minimize the impact of the waves, thereby providing safe environment for ships and increasing overall efficiency.

The first record of using floating structure as a breakwater dates back to the early $19^{\text {th }}$ century. In 1811 , General Bentham, the Civil Architect and Principal Surveyor of the Royal Navy of Great Britain, proposed a breakwater model for the British fleet at Plymouth. The breakwater consisted of triangular sections of floating wood frames moored with iron chains. Although, its cost was about one-tenth the finally adopted rubble and granite mound structure, the idea was rejected due to concerns about its effectiveness during severe storms (Ozeren, 2009; Tu et al. 2008).

Simulation of laboratory conditions with numerical models offers means to study and understand the physical model more effectively. Several types of numerical models and software development platforms are available for simulation of waves. Paddle wave maker with CFD is one such simulation tool, which uses a discrete approach based on finite volume (Panahi

Corresponding author: A. Najafi-Jilani

e-mail:a.jilani@iiau.ac.ir et al. 2010). In this paper, two-dimensional simulations were made using the Paddle Wave maker with Smoothed Particle Hydrodynamic (SPH) method.

SPH method is the oldest mesh less particle method, developed independently by Monaghan (1994) to simulate the asymmetry problems in astrophysics and used to predict the motion of separated particles with respect to time. SPH method using Lagrangian technique has been used successfully for simulation of free surface hydrodynamics. As examples, it has been used for surface flows including waves in the smooth beaches, plunging wave breaker and simulation of dam failure. It has also shown high potential for simulation of a wide range of numerical models such as coastal waves, wave form of landslide, wave impact on structures and dams etc.

The broad objective of the present study is to simulate the interactions of waves of varying height and period with the floating breakwater structure using the SPH method.

\section{MATHEMATICAL FORMULATION}

The general formulation of any function can be represented by an integral equation of the following form(Dalrymple, 2007):

$f(x)=\int_{\Omega} f\left(x^{\prime}\right) \delta\left(x-x^{\prime}\right) d x^{\prime}$

where, $\mathrm{f}$ is the function of the position vector $\mathrm{x}, \Omega$ is the integral volume which $\mathrm{x}$ encompasses, and $\delta(\mathrm{x}-\mathrm{x}$ ') is the Dirac delta function, which is defined as follows: 
$\delta\left(x-x^{\prime}\right)= \begin{cases}1, & x=x^{\prime} \\ 0, & x \neq x^{\prime}\end{cases}$

If $\mathrm{f}(\mathrm{x})$ in domain $\Omega$ on the right side of equation (1) is defined, the exact value of the function $\mathrm{f}(\mathrm{x})$ can be obtained. By substituting the Dirac delta function with the smoothing kernel function, smoothing function, or in short kernel W (x$\left.x^{\prime}, h\right), f(x)$ takes the following form:

$$
<f(x)>=\int_{\Omega} f\left(x^{\prime}\right) \mathrm{W}\left(x-x^{\prime}, h\right) d x^{\prime}
$$

where, $\mathrm{h}$ represents the smoothing length or influence length of $\mathrm{W}$ function, and specifies the range of integral. The kernel function becomes zero outside this area.

In the SPH method, the continuous fluid environment is separated into a set of discrete elements (referred to as particles). The physical quantity of any particle can be obtained by summing the relevant properties of all the particles which lie within the range of integral the kernel approximation function. The integral form can be converted to series form if $\mathrm{dx}^{\prime}$ in particle space is converted to finite particle volume $\left(\Delta V_{j}\right)$. The particle mass $\left(m_{j}\right)$ can then be estimated by the following equation:.

$\boldsymbol{m}_{j}=\Delta \boldsymbol{V}_{j} \rho_{j}$

where, $\rho_{j}$ is the density of particle $j$. The discrete form of the equation can be represented by:

$\left(\frac{d \mathrm{u}}{\boldsymbol{d} t}\right)_{i}=-\frac{1}{\rho_{i}} \sum_{j} \frac{\boldsymbol{m}_{j}}{\rho_{j}} \boldsymbol{p}_{j} \nabla_{i} \mathrm{~W}_{i j}-\boldsymbol{g}$

where, $\mathrm{N}$ is the number of particles. The final form of the equation can be represented by:

$<\boldsymbol{f}(\boldsymbol{x})>=\sum_{j=1}^{N} \frac{\boldsymbol{m}_{j}}{\rho_{j}} \boldsymbol{f}\left(\boldsymbol{x}^{\prime}\right) \cdot \mathrm{W}_{i j}$

The interpolation of smoothing kernel function plays a key role in SPH method. Generally, kernel of three point is important:

- estimation of pattern and interpolation of field function,

- setting dimensions of field effect,

- accurate and consistent interpolation in discrete state (approximate kernel).

$$
\mathrm{W}(\boldsymbol{r}, \boldsymbol{h})=\alpha_{\boldsymbol{D}}\left\{\begin{array}{cc}
1-\frac{3}{2} \boldsymbol{q}^{2}+\frac{3}{4} \boldsymbol{q}^{3} & 0 \leq \boldsymbol{q} \leq 1 \\
\frac{1}{4}(2-\boldsymbol{q})^{3} & 1 \leq \boldsymbol{q} \leq 2 \\
0 & \boldsymbol{q} \geq 2
\end{array}\right.
$$

In the present study, cubic kernel function of the following form has been used for the simulation (7).

Various parameters in the above equation are defined as follows:

$r=x-x_{j}, \quad q=\frac{r}{h}, \quad \alpha_{D}=\frac{10}{7 \pi h^{2}}$

An example of a kernel approximation is shown in Fig. 1.

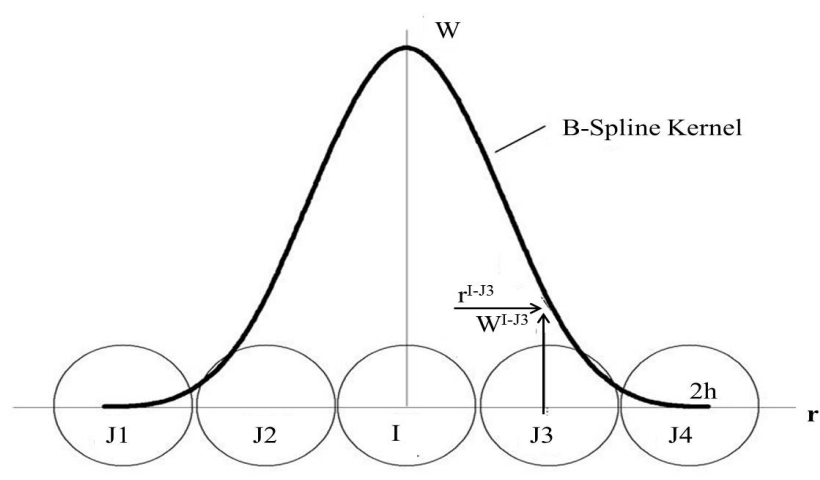

Density at $\mathrm{I}, \rho^{\mathrm{I}}=\mathrm{m}^{\mathrm{J} 1} \mathrm{~W}^{\mathrm{I}-\mathrm{J} 1}+\mathrm{m}^{\mathrm{J} 2} \mathrm{~W}^{\mathrm{I}-\mathrm{J} 2}+\mathrm{m}^{\mathrm{I}} \mathrm{W}^{\mathrm{I}-\mathrm{I}}$ $+\mathrm{m}^{\mathrm{J} 3} \mathrm{~W}^{\mathrm{I}-\mathrm{J} 3}+\mathrm{m}^{\mathrm{J} 4} \mathrm{~W}^{\mathrm{I}-\mathrm{J} 4}$

(a) One-dimensional.

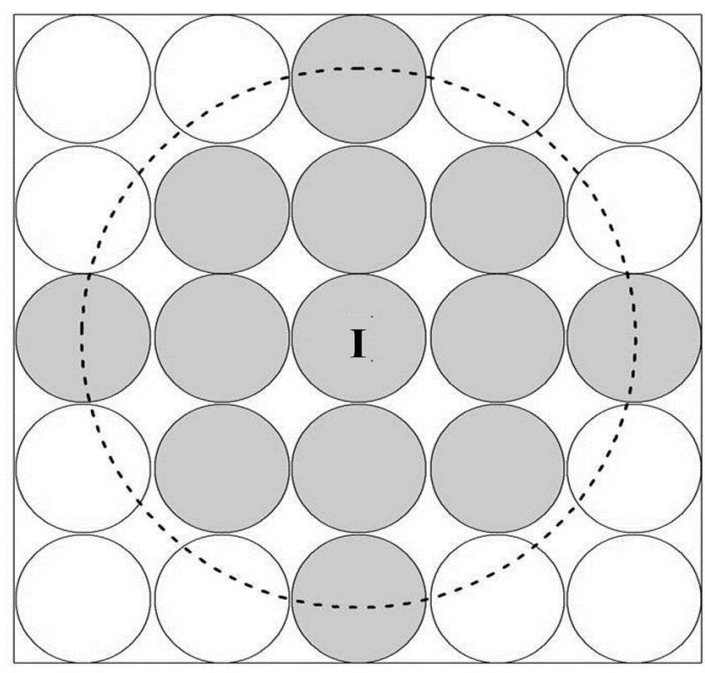

(b) Two-dimensional.

Fig. 1 An example of kernel function (Lo and Shao, 2007).

Basic equations of fluid dynamics for three physical principles of conservation mass, momentum and energy are defined. These equations can be used in different forms depending on the circumstances, for solving the problem. Two common forms of the equation for problem solving are Eulerian and Lagrangian. The general form of the continuity equation is given by:

$\frac{1}{\rho} \frac{\boldsymbol{d} \rho}{\boldsymbol{d} t}=-\nabla \cdot \boldsymbol{u}$ 
where, $u$ is the speed and $\rho$ is the fluid density. Equation (8) in series form is expressed by equation (9) as follows:

$$
\left(\frac{1}{\rho} \frac{\boldsymbol{d} \rho}{\boldsymbol{d} \boldsymbol{t}}\right)_{i}=\sum_{j} \frac{\boldsymbol{m}_{j}}{\rho_{j}} \mathrm{u}_{j} \cdot \nabla \mathrm{W}_{i j}
$$

where, $W_{i j}=W\left(x_{i}-x_{j}\right)$. Gradient kernel for second particle (j) is equal the negative gradient kernel for the first particle (i). The Lagrangian form of momentum equation is expressed as:

$$
\begin{aligned}
& \boldsymbol{B}=\frac{\boldsymbol{c}_{0}^{2} \rho_{0}}{\gamma} \\
& \frac{\boldsymbol{d u}}{\boldsymbol{d} \boldsymbol{t}}=-\frac{1}{\rho} \nabla \boldsymbol{p}-\boldsymbol{g}
\end{aligned}
$$

where, $g$ is the acceleration due to gravity, and $\mathrm{p}$ is the pressure.

Momentum equation in the form of the SPH method is shown in equation (11).

$$
\left(\frac{d \mathrm{u}}{d t}\right)_{i}=-\frac{1}{\rho_{i}} \sum_{j} \frac{\boldsymbol{m}_{j}}{\rho_{j}} \boldsymbol{p}_{j} \nabla_{i} \mathrm{~W}_{i j}-g
$$

Regardless of viscosity, instability develops in some phenomena modeling with SPH method. Boiling is one of these forms of instability which make the particles to move irregularly(Monaghan, 1999). In the present study, since laminar viscosity is considered, the momentum equation changes to the following form:

$$
\frac{\boldsymbol{d u}}{\boldsymbol{d} \boldsymbol{t}}=-\frac{1}{\rho} \nabla \boldsymbol{P}+\boldsymbol{g}+v_{0} \nabla^{2} \boldsymbol{u}
$$

In the above equation, $v_{0}$ is the kinematics viscosity in laminar flow and its value is $10^{-6} \mathrm{~m}^{2} \cdot \mathrm{s}^{-1}$. The viscosity term in the SPH method is written as follows (Dalrymple, 2007).

$v_{0} \nabla^{2} \overrightarrow{\boldsymbol{u}}=\sum \boldsymbol{m}_{\boldsymbol{b}}\left(\frac{4 v_{0} \overrightarrow{\boldsymbol{r}} \nabla \boldsymbol{W}_{i j}}{\left(\rho_{\boldsymbol{i}}+\rho_{\boldsymbol{j}}\right)|\overrightarrow{\boldsymbol{r}}|^{2}}\right) \overrightarrow{\boldsymbol{u}}$

\section{NUMERICAL MODELING}

The fluid in the SPH formalism is assumed as weakly compressible. This facilitates the use of an equation of state to determine fluid pressure, which can be solved much faster than the Poisson's equation. However, the compressibility is adjusted to lower the speed of sound so that the time step in the model (using a Courant condition for the speed of sound) is reasonable. Another limitation on the compressibility is imposed by the requirement that sound speed should be about ten times faster than the maximum fluid velocity, thereby keeping density variations to less than $1 \%$.

Following relationship between pressure and density is assumed to follow the expression (Monaghan and Kos, 1999).

$$
\boldsymbol{p}=\boldsymbol{B} \rho_{s}\left[\left(\frac{\rho}{\rho_{s}}\right)^{\gamma}-1\right]
$$

where, $\mathrm{g}=7, B=\frac{\boldsymbol{c}_{0}^{2} \rho_{0}}{\gamma}, \rho_{\mathrm{o}}=$ reference density $(1000 \mathrm{~kg}$ m-3), and $\boldsymbol{c}_{0}=\boldsymbol{c}\left(\rho_{0}\right)=\left.\sqrt{(\partial P / \partial \rho)}\right|_{\rho_{0}}$ the speed of sound at the reference density.

\section{BOUNDARY CONDITIONS}

Unlike mesh-based methods such as finite element and finite difference, the boundary conditions for mesh less modeling methods such as SPH isn't very straightforward. SPH method boundary conditions suffer from lack of consistency in the continuous approximation requiring special measures to simulate these areas. For modeling, a fixed set of virtual particles is used on the fluid interface and solid surface (Monaghan and Kos, 1999). The function of these virtual particles is to create a force in the opposite direction to particles moving close to each other to prevent water particles from crossing a solid boundary. The particles constituting the boundary exert central forces, analogous to intermolecular forces on the fluid particles. Free surface is detected by computing the density of particles. Weighted interpolation employed in SPH method indicated a decreased density outside the free surface, suggesting absence of particles. If the density is less than $1 \%$ of its value for the internal fluid particles, the particle component is considered as free surface. It is required to compare the laboratory experimental data with the numerical simulation results to verify the model. As the simulated Paddle Wave maker only has the ability to make regular waves, it can be used for generating waves from fundamental principles of regular wave theory. Fig. 2 shows an example of simulated Paddle Wave maker generated using SPH method.

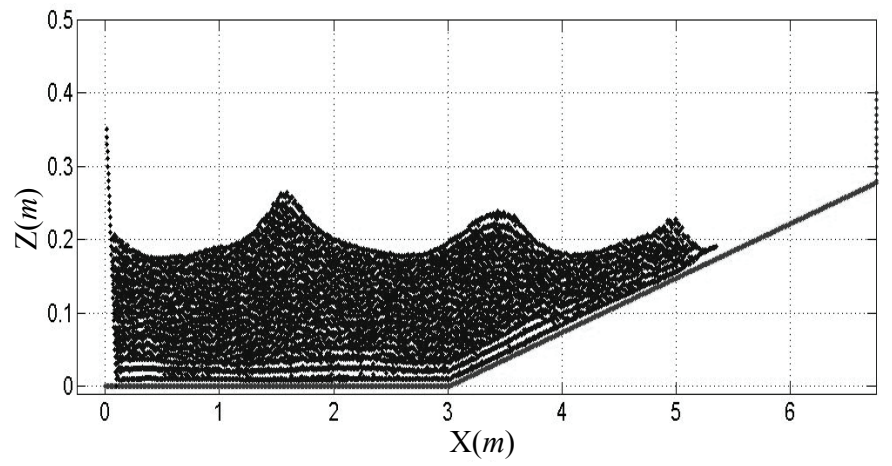

Fig. 2 Image of Paddle wavemaker simulation by SPH method $($ time $=4.9502 s$, frame $=99)$. 
To verify the results, the surface profiles are first obtained with the theoretical values and compared with the SPH simulation. Following the theories of wave maker, the ratio of wave height to stroke of Paddle wave maker are obtained by:

$$
\frac{\boldsymbol{H}}{\boldsymbol{S}}=\frac{4 \sinh \boldsymbol{k} \boldsymbol{h}}{\sinh 2 \boldsymbol{k} \boldsymbol{h}+2 \boldsymbol{k h}}\left(\sinh \boldsymbol{k} \boldsymbol{h}+\frac{1-\cosh \boldsymbol{k} \boldsymbol{h}}{\boldsymbol{k} \boldsymbol{h}}\right)
$$

where, $\mathrm{H}$ is the wave height, $S$ is the wave stroke, $k$ is the wave number ( $=2 \pi / L$ where $L$ is the wavelength), and $h$ is the water depth. Fig. 3 shows the image of a Paddle wave maker.

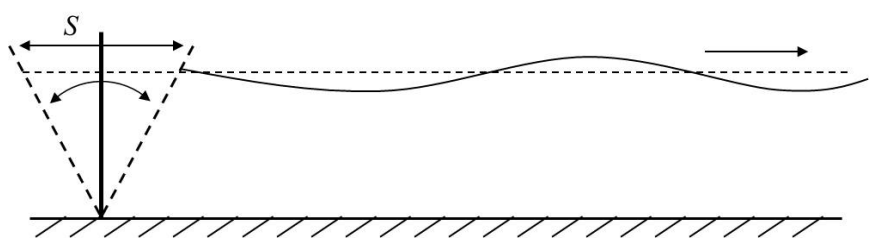

Fig. 3 Schematic picture of Paddle wave maker (flap type).

\section{RESULTS AND DISCUSSIONS}

Due to lack of laboratory data for Paddle wave maker, we used the data available in the literature (Dalrymple, 2007). For comparison of data, a second wave period of $5 \mathrm{~cm}$ wave height and $40 \mathrm{~cm}$ depth of water was considered. The results of SPH numerical model showed good agreement with the experimental data (Fig. 4).

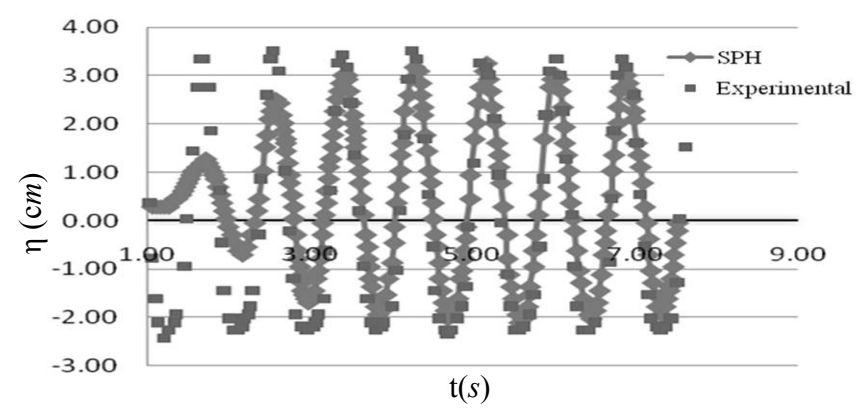

Fig. 4 Comparison of water surface profiles in laboratory data and SPH model developed in this research.

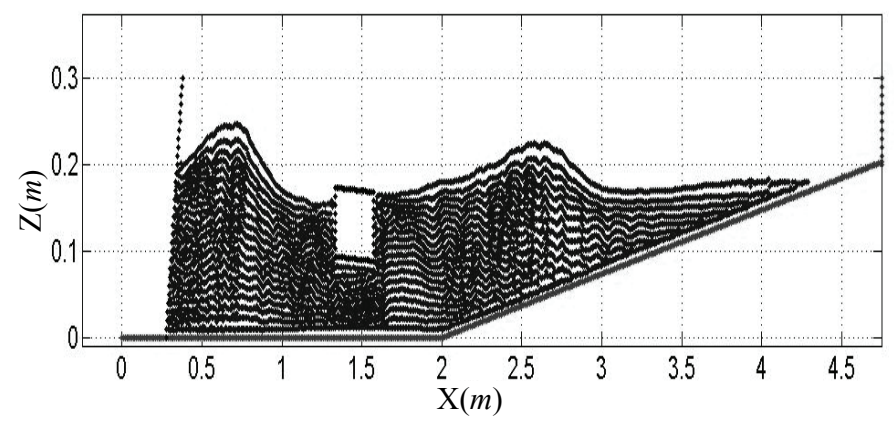

Fig. 5 Modelling of floating structure with SPH method developed in this research (time $=2.7502 \mathrm{~s}$, frame $=55$ ).

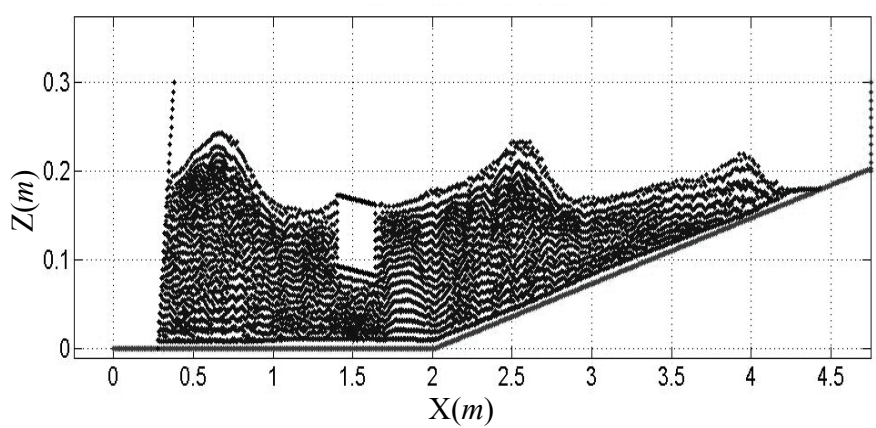

Fig. 6 Modelling of floating structure with SPH method developed in this research $($ time $=6.9502 s$, frame $=139)$.

Until now, the research on simulation of floating breakwater has focused only on the simulation of floating structures on water level. Accurate information related to wave height behind the floating structures, and motion of thefloating structure is not available. Hence, this study has paid attention to simulation of floating structure and motion in regular waves. Fig. 5 and 6 show the motion of floating structures in two different time steps.

\section{CONCLUSION}

SPH technique was applied to simulate floating breakwater and its interaction with the waves. Results of numerical modeling made with SPH are in good agreement with extant theory and experimental data. Hence, SPH method is a good tool for modeling and simulating experimental conditions. The simulation error rate is insignificant, and can be ignor

ed. Hence the technique can be a successfully applied for numerical simulation of not only breakwater floating structures, but also many other simulations.

\section{REFERENCES}

Dalrymple, R.A., 2007. Particle Methods and Waves, with emphasis on SPH. Publications of Johns Hopkins University, 310, pp.1-32.

Lo, E.Y.M. Shao, S.D., 2007. Simulation of near-shore solitary wave mechanics by an incompressible SPH method, Applied Ocean Research, Vol. 24. pp. 275-286.

Monaghan, J.J., 1994. Simulating free surface flows with SPH. Journal of Computational Physics, 10(2), pp. 399-406.

Monaghan, J.J. Kos, A., 1999. Solitary waves on a Cretan beach. J Waterway, Port, Coastal and Ocean Eng; 125(3). pp. $145-154$.

Panahi, R. Shafie, far. M. and Salehi, A.A., 2010. Simulation of Paddle wave maker with CFD. 9th Iranain Hydraulic conferences.(in Persian).

Ozeren,Y., 2009. Experimental and Numerical Investigations of Floating Breakwater Performance. Ph.D. thesis. University of Mississippi.

Tu, J. Yeoh, G.H. Liu, C., 2008. Computational Fluid Dynamics: A Practical Approach. Elsevier. 